\title{
Fast responsive, optical trace level ammonia sensor for environmental monitoring
}

\author{
Tobias Abel, Birgit Ungerböck, Ingo Klimant and Torsten Mayr
}

\begin{abstract}
Background: Ammonia is a ubiquitous chemical substance which is created in technical and biological processes and harmful to many different organisms. One specific problem is the toxicity of ammonia in fish at levels of $25 \mu \mathrm{g} / \mathrm{l}$ - a very common issue in today's aqua culture. In this study we report a development of a fast responsive, optical ammonia sensor for trace concentrations.

Results: Different hydrogels have been investigated as host polymers for a pH based sensing mechanism based on fluorescent dyes. A porous hydrophobic fluoropolymer membrane was used as an ion barrier cover layer to achieve a good ammonia permeability. The sensor's sensitivity towards ammonia as well as crosssensitivity towards $\mathrm{pH}$-value and salinity, and the temperature dependency have been determined. Two different methods to reference fluorescence signals have been employed to eliminate intensity-based measurement drawbacks.

Conclusion: The presented sensor features high sensitivity and a fast response even at concentrations near 1 ppb. No cross sensitivity towards $\mathrm{pH}$ and salinity could be observed and temperature dependency was determined as compensateable. Both referencing approaches prove themselves to be able to provide a simple use of the sensor for in-field applications.
\end{abstract}

Keywords: Optical sensor, Ammonia sensor, Fluorescence sensor, Fish farming, Aqua culture, Dual lifetime referencing, Two wavelength ratiometric referencing

\section{Background}

Ammonia is a very widespread chemical in our world. It is not only present in substances like refrigerants, household cleaners, and (most abundantly) industrial fertilizers, but is also produced in nature by all animal cells [1] resulting from degradation of amino acids [2], food putrefaction [3], excretion, and decomposition of waste and sewage. Due to this broad use and existence of ammonia it can be found in the atmosphere [4], the soil [5], as well as in river- [6] and seawater [7]. Furthermore ammonia is toxic to any kind of animals, from microorganisms [8] to more differentiated life forms [9]. These circumstances lead to an increasing demand for robust, cheap and continuous means of measuring ammonia in environmental monitoring, food processing and medical applications [10]. This study approaches the problem of ammonia monitoring in the aquatic habitat. The developed sensor is suitable for the application in fish farming

\footnotetext{
* Correspondence: torsten.mayr@tugraz.at

Institute of Analytical Chemistry and Food Chemistry, Graz University of Technology, Stremayrgasse 9, Graz 8010, Austria
}

[11], which itself is an attempt to lessen global overfishing while providing fish for global demands.

Many different methods have been employed for analytical detection of ammonia, such as flow spectrometrics [12], potentiometric electrodes [13], IR absorption [14], amperometric [6] and conductivity [15] measurements, or spectrophotometric approaches based on the Berthelot reaction [16] or on Nessler's method [12]. However, these methods consume chemicals, need batch separation from the analytical sample, or require sample pretreatment and/ or expensive instrumentation, which prevent these approaches being applied for continuous and simple monitoring tasks. Optical ammonia sensors [17-19] based on $\mathrm{pH}$-indicators can fulfill these conditions, since they can be manufactured for low-cost in high quantities and used with simple instrumentation without sample pretreatment.

In this study we present an optical ammonia sensor based on commercially available materials, simple in both manufacturing and operation, designed to fulfill ammonia monitoring functions as demanded in modern 
fish farms. The ammonia sensitive layer consists of a hydrogel in which two non-sensitive, fluorescent dyes and a non-fluorescent $\mathrm{pH}$-indicator are entrapped. The two fluorescent dyes and the indicator form a Förster resonance energy transfer [20] (FRET) cascade due to their overlapping emission and absorption spectra of the FRET donor and acceptor respectively. The $\mathrm{pH}-$ indicator is protonated in absence and deprotonated in presence of ammonia, changing its absorption spectra and quenching the FRET emission in the presence of ammonia. The analytical information is gained by measuring the cascade's fluorescence intensity. To overcome problems of varying fluorescence intensities derived from differing layer thicknesses and power attenuation by the instrumentation we employed two different reference methods, one of which even allows ratiometric imaging techniques using the color channels of a CCD camera [21].

\section{Results and discussion}

The general structure of an optical ammonia sensor is based on a hydrophilic polymer, in which the sensing chemistry is immobilized, entrapped sandwich-like between a supporting material and a proton barrier, which permits a permeation of ammonia but prohibits a penetration of protons into the host material.

\section{Choice of indicators and dyes}

The main characteristic which defines the dynamic range of an ammonia sensor based on $\mathrm{pH}$ indicators is the $\mathrm{pK}_{\mathrm{a}}$ of said indicator. Ammonia, which migrates into the host material, deprotonates the indicator and forms an ammonium salt with the indicator as counter ion, as described by equation 1 . Though there are some ammonia sensors based on fluorescent indicators, [22-24], the majority rely on absorbance-based $\mathrm{pH}$ indicators $[17,25-28]$, in a broader $\mathrm{pK}_{\mathrm{a}}$ range available.

$$
\mathrm{NH}_{3}+\mathrm{IndH} \rightleftharpoons \mathrm{NH}_{4}^{+}+\mathrm{Ind}^{-}
$$

The used indicator was bromophenol blue (BPB) with a $\mathrm{pK}_{\mathrm{a}}$ of 4.1 [17]. The indicator shows different absorbance spectra within the visible field. The deprotonated form shows an absorbance maximum at $600 \mathrm{~nm}$ while the protonated form shows an absorbance maximum at $425 \mathrm{~nm}$ (see Figure 1 for normalized spectra). Thus, absorbance carries the analytical information. However, absorbance cannot be as easily measured (e. g. by reflection in thin polymer films) as fluorescence. For this reason two more dyes were introduced, which form a FRET cascade: a donor (Coumarin 545T or "C545T"), an acceptor (Macrolex Fluorescent Red G or "MFR") and a quencher (BPB). The donor is excited and transfers its
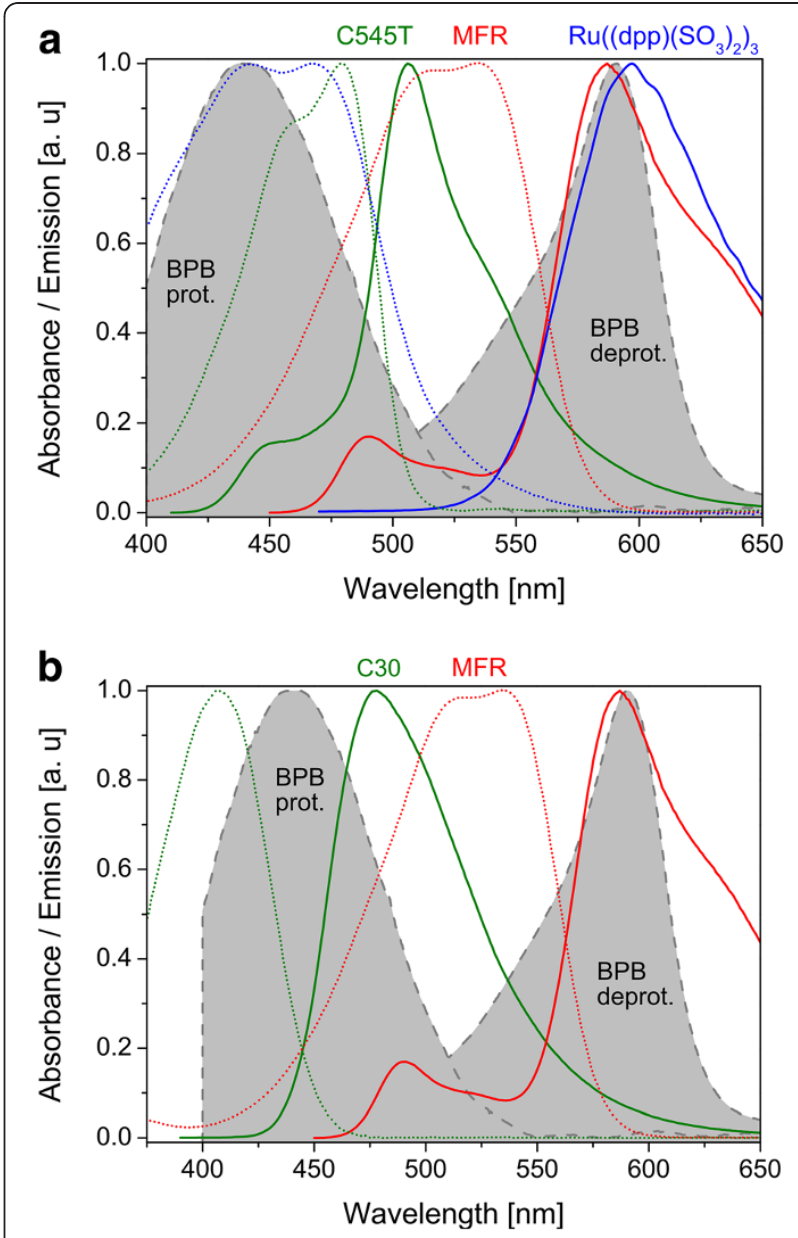

Figure 1 Absorbance and emission spectra of used sensor compositions. a shows the DLR system: absorbance (dotted line) and emission (solid line) spectra of Coumarin 545T (green), Macrolex Fluorsecent Red (red) and the Ruthenium complex (blue) as well as the absorbance spectra for the protonated and deprotonated forms of bromophenol blue (grey areas). b shows the TWR system: absorbance (dotted line) and emission (solid line) spectra of Coumarin 30 (green), Macrolex Fluorsecent Red (red) as well as the absorbance spectra for the protonated and deprotonated forms of bromophenol blue (grey areas).

energy to the acceptor dye, emitting subsequently light at $600 \mathrm{~nm}$. This is prevented, if in presence of ammonia the indicator's deprotonated form is within the membrane, because the energy will then be passed from MFR onto the indicator and the emission diminishes. This approach offers additional advantages; due to the excitation by energy transfer, photo bleaching of the $\mathrm{pH}$ indicator can be minimized. Furthermore, high intensities can be obtained by an increased donor concentration, increasing the absorbance of excitation light [29].

The indicator and dyes necessary for the different referencing techniques are discussed within the appropriate sections. 


\section{Choice of polymers}

There are some important properties a polymer has to fulfill to be considered as host material for an ammonia sensor: The host polymer has to stabilize both the hydrophilic ammonium and the hydrophobic protonated indicator, which means that the polymer's hydrophilicity is a key factor for sensitivity and stability of the sensor. Furthermore the polymer must not have alkaline properties, so that the protonated form of the $\mathrm{pH}$ indicator is stable inside the membrane.

Cellulose esters have been used as host polymers $[23,24]$, since they fulfill these requirements. Hydrogels even surpass these characteristics. Their water absorption $(70,50$ and $30 \%$ for HydroMed D1, D4 and D7 respectively [30] and their strong adhesion properties make them a very suitable choice. As seen in Figure 2a these hydrogels outperform cellulose acetate (CA) in terms of detectable concentration ranges. This is a very crucial requirement, since ammonia displays toxicity towards aquatic and amphibic organisms at concentrations of even $25 \mu \mathrm{g} / \mathrm{l}$. CA shows $50 \%$ of the maximum intensity decrease at about $90 \mu \mathrm{g} / \mathrm{l}$ ammonia, the hydrogels D7, D4 and D1 undermatch this at concentrations of 13, 1 and $2 \mu \mathrm{g} / \mathrm{l}$ respectively. This shows that the sensitivity of the same sensing chemistry is higher in hydrogels than in CA.

\section{Choice of proton barrier}

Ammonia sensors based on a $\mathrm{pH}$ sensitive layer require a proton barrier for liquid samples to prohibit a response to the $\mathrm{pH}$ of the tested sample. Different materials have been used; silicon being the probably most widespread reported one $[23,24,26]$. The proton barrier has to feature two properties: impermeability for protons and permeability for ammonia. Facing minimal concentrations during trace measurements, reassuring a high permeability is of the utmost importance to keep the sensors' response time within reasonable limits.

In the study presented a PTFE-based membrane filter has been used, displaying not only the strong hydrophobic properties of technical fluoropolymers, but also an extraordinary permeability for ammonia [31,32] due to its highly porous structure [33]. Moreover the white, reflective filter surface offers the possibility to keep the analyte sensitive layer thickness below two micro meter whilst maintaining a sufficiently high signal level and minimizing response times. Moreover fluoropolymers offer a high resistivity against biofouling and thus the establishment of biofilms on the sensor's surface [34], which can lead to deterioration in the sensor performance.

\section{Sensor response times}

Due to the filter membranes used, and the thin polymer layer it was possible to achieve very short response
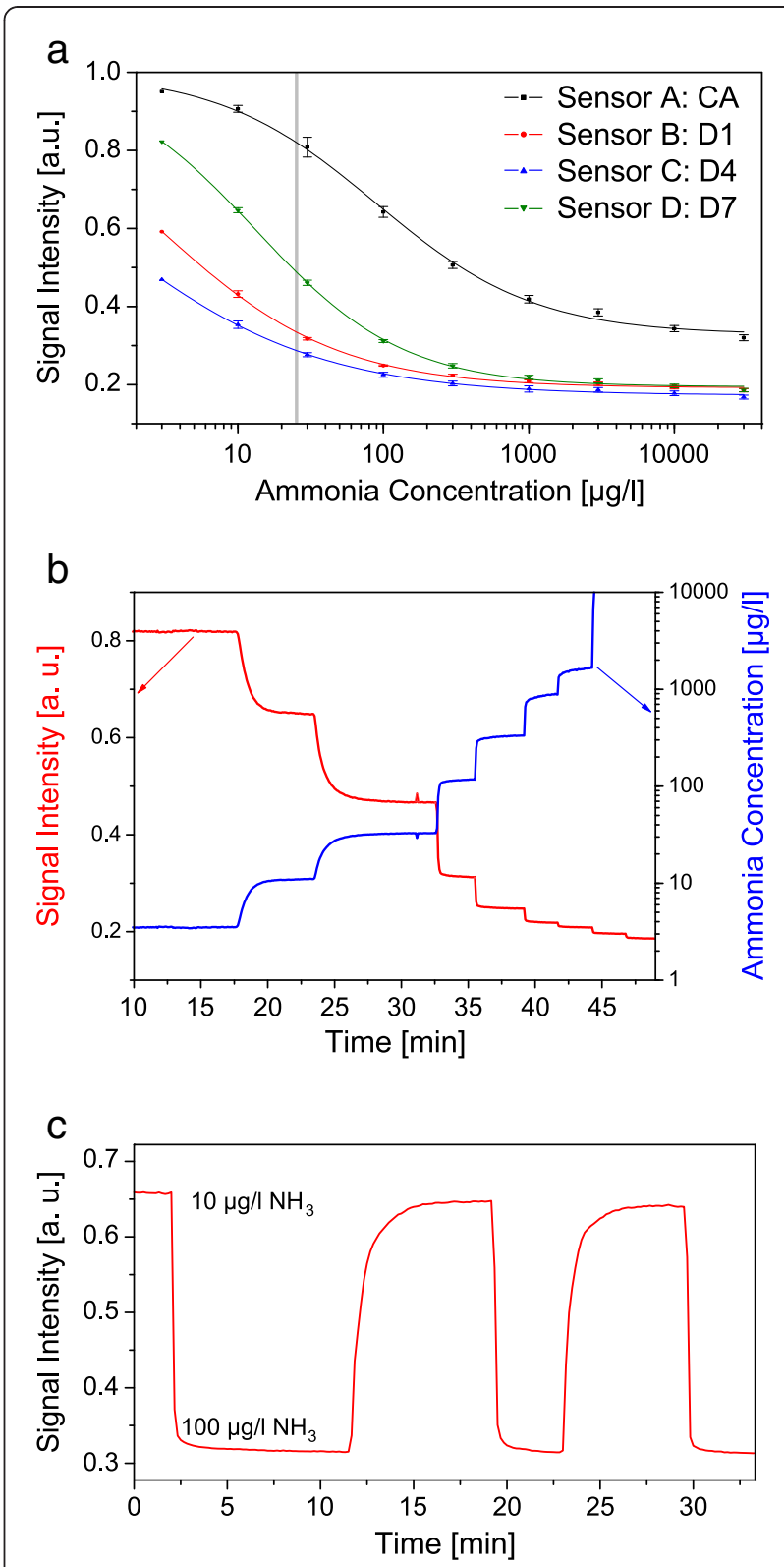

Figure 2 Sensitivity and response of the ammonia sensor. (a) Calibration plots for ammonia sensors A, B, C, and D in a concentration range between 3 and 30,000 $\mu \mathrm{g} / \mathrm{l}$ with a view to the ammonia toxicity level in fish of $25 \mu \mathrm{g} / \mathrm{l}$ (grey line) (mean values from 6 sensor layers); (b) time resolved signal response (red graph) and calculated concentrations (blue graph) based on sensor B; (c) response and recovery response of sensor B between 10 and $100 \mathrm{\mu g} / \mathrm{l}$ ammonia in $100 \mathrm{mM}$ phosphate buffer at $\mathrm{pH}$ 7.2.

times. The response time $t_{90}$ for a change between $3 \mu \mathrm{g} / \mathrm{l}$ and $10 \mu \mathrm{g} / \mathrm{l}$ is about 120 seconds (see Figure $2 \mathrm{~b}$ ). The $t_{90}$ response time and the recovery response time between $10 \mu \mathrm{g} / \mathrm{l}$ and $100 \mu \mathrm{g} / \mathrm{l}$ were determined to be 60 and 50 seconds respectively (see Figure 2c), decreasing with higher concentrations down to 20 seconds. This sensor clearly outperforms silicon as proton barrier, of which $t_{90}$ 
response times of 30 [26], 40 [23], even 70 [35] minutes have been reported.

The sensors employing hydrogels (sensor B, C and D) displayed slightly shorter response times between $10 \mu \mathrm{g} / \mathrm{l}$ and $100 \mu \mathrm{g} / \mathrm{l}$ (60, 60 and 50 seconds respectively) than the same setup using cellulose acetate (sensor A, 90 seconds), which demonstrates that the ammonia permeability is in hydrogels higher than in cellulose acetate.

\section{Cross sensitivity and dependencies}

Different tests were carried out to assess the sensors' cross sensitivity. The impermeability towards protons is demonstrated as seen in Figure 3a. No change of signal intensity could be registered, even with $\mathrm{pH}$ buffers far beyond the $\mathrm{pK}_{\mathrm{a}}$ value of the used indicator.

Also the sensor's cross sensitivity towards varying salinities was evaluated (see Figure 3b). Taking into account that the ammonia-ammonium equilibrium changes with salinity (which can be mathematically compensated [36]), the sensor shows virtually no divergence in its calibration in ammonia solutions containing $3.5 \%(w / w)$ sodium chloride compared to sodium chloride free solution.

There is a specific dependency in terms of temperature. Figure 3c show three different calibrations, all carried out with the identical sensor and buffers. The ammonia concentration was corrected for the shifted ammonia-ammonium equilibrium (see experimental section, equation 3). Still, a slight dependency towards temperature was measured within the relevant concentrations. This can be attributed to the universal characteristic of increased fluorescence at lower temperatures and a temperature dependency of the indicator's pKa. Compensation can be carried out by a simultaneous measurement of the temperature. Dual analyte- temperature sensor where recently published by Borisov et al. [37]. An accurate temperature measurement is mandatory due to the temperature dependency of the ammonia/ammonium equilibrium.

\section{Dual-Lifetime Referencing (DLR)}

All measurements so far discussed are intensity based; those measurements are error-prone in real-world applications. These errors can result from manufacturing inhomogenities, different alignments, or varying performances of read-out units. Therefore, we investigated the application of two different signal referencing methods to overcome the limitations of intensity measurements to obtain a universal and user friendly system suitable for field measurements.

The first reference method employed is Dual Lifetime Referencing [22,38], (DLR). This method requires a phosphorescent reference dye featuring a long decay time, equal excitation and emission wavelengths as the

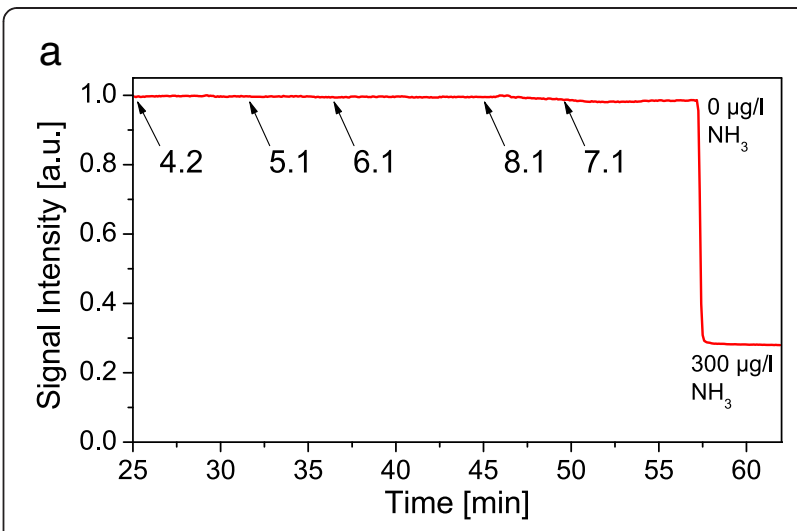

b

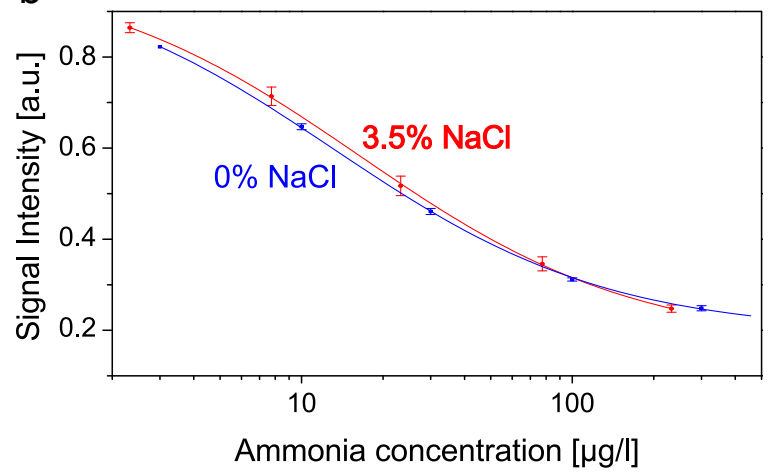

C

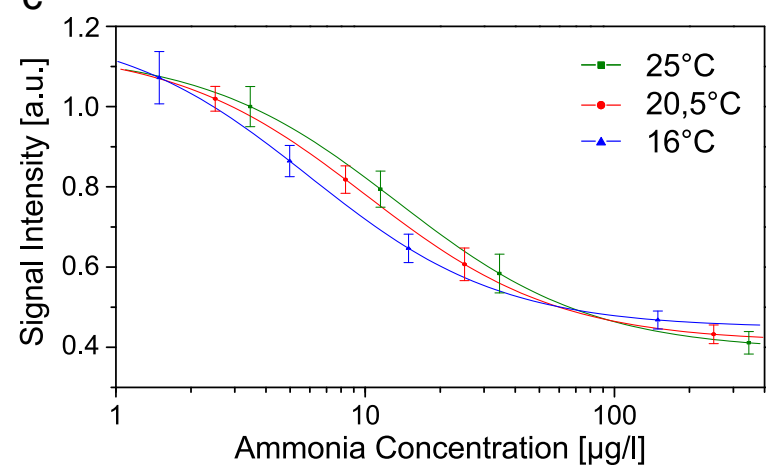

Figure 3 Cross sensitivity of sensor $B$ towards $\mathrm{pH}$ (a) and salinity (b, mean value of 4 sensor foils), and temperature dependency (c, mean value of 4 sensor foils).

sensing chemistry and no sensitivity towards the analyte or other substances. Silica particles were chosen as host material due to their simple synthesis, their insolubility in organic solvents, and their low oxygen permeability. These particles contained a phosphorescent Ruthenium complex, which would be an oxygen-sensitive probe unless incorporated inside the gas-impermeable spheres. These spheres are evenly dispersed in the sensor cocktail during the manufacturing process and homogenously distributed in the host polymer. Furthermore, it was necessary to decrease the sensors brightness (compared 
to intensity measurements) by reducing the FRET-pair donor's concentration to match the luminescence intensities of the sensing chemistry and the reference.

The measurement is carried out with a sinusoidally modulated light source, which excites the indicator and the reference particles. The detected signal exhibits a high or low phase shift, depending on the ratio of the intensities of the reference and the indicator. Figure $4 \mathrm{a}$ depicts the calibration curve of the presented DLRreferenced sensor. The red graph shows the measured phase shifts. The blue graph shows the calculated cotangent values, which is directly proportional to the indicators intensity. It has to be remarked, that the high deviation at $3 \mu \mathrm{g} / \mathrm{l}$ is not originated by measurement problems, but is derived from the steepness of the cotangent functions at values next to zero.

Figure $4 \mathrm{~b}$ shows a long term stability test carried out for 6 days using two different methods: Both sensor batches were stored in a buffer containing $100 \mu \mathrm{g} / \mathrm{l} \mathrm{am}$ monia, and characterized on the first and sixth day of storage. One batch was stored in complete darkness while the other batch was illuminated (6 samples per minutes over the period of 6 days equals 8600 measurement points) to investigate if the drop in signal was caused by photo bleaching. Both tested sensor batches show a drop of the cotangent-values at low ammonia concentrations after 6 days while maintaining the signal values at higher concentration. Since both sensor calibrations drop the same amount it is assumed, that the sensor itself is not prone to photo bleaching but may suffer from leaching or migration problems due to incomplete encapsulation of the indicator and reference dyes. Despite this change in sensor characteristics a recalibration and further use of the tested sensors is still possible. However, for long-time measurements we would recommend an exchange of sensor foils at regular intervals.

\section{Two Wavelength Ratiometric (TWR)}

A second referencing method was employed; based on ratiometric measurement of two different emission intensities recorded at two different bands of wavelength. The sensing chemistry was modified to gain two different emission peaks with sufficient difference in wavelength and comparable ranges of emission intensity over the whole range of tested ammonia concentrations. The previously used FRET donor, Coumarin 545T, was exchanged for Coumarin 30 (C30), a dye similar in absorbance and quantum yield but featuring shorter absorption and emission wavelengths, which was crucial to obtain two distinctly separated emission peaks. The reduced overlap of the $\mathrm{C} 30$ emission and the acceptors absorption spectra (MFR) leads to an decreased FRET efficiency and noticeable emission of the donor in the region of $470 \mathrm{~nm}$ (see Figure 5a).

This FRET cascade is combined with the BPB indicator forming the ammonia sensing system. BPB shows two different absorbance bands, its protonated form overlaps the donor's (C30) emission and the deprotonated form overlaps the acceptor's (MFR) emission. This leads to an opposing change in intensities with ascending ammonia concentrations, namely an increasing emission of the donor and a decreasing emission of the acceptor (see Figure 5a). The signal can be referenced by dividing the intensity of the $625 \mathrm{~nm}$ peak by the intensity of the $470 \mathrm{~nm}$ peak, either mathematically from a spectrum (see Figure 5a), by a measurement with two different band pass filters or by imaging techniques with a color camera (see Figure 5b).

For the "two filters approach" a lock-in amplifier was used, connected with a 405 nm LED, a trifurcated fiber bundle and two PMT tubes with two different band pass filters for each emission peak. The emission intensity of both peaks was measured and referenced by dividing the red by the blue channel. Figure $5 \mathrm{c}$ depicts a longtime
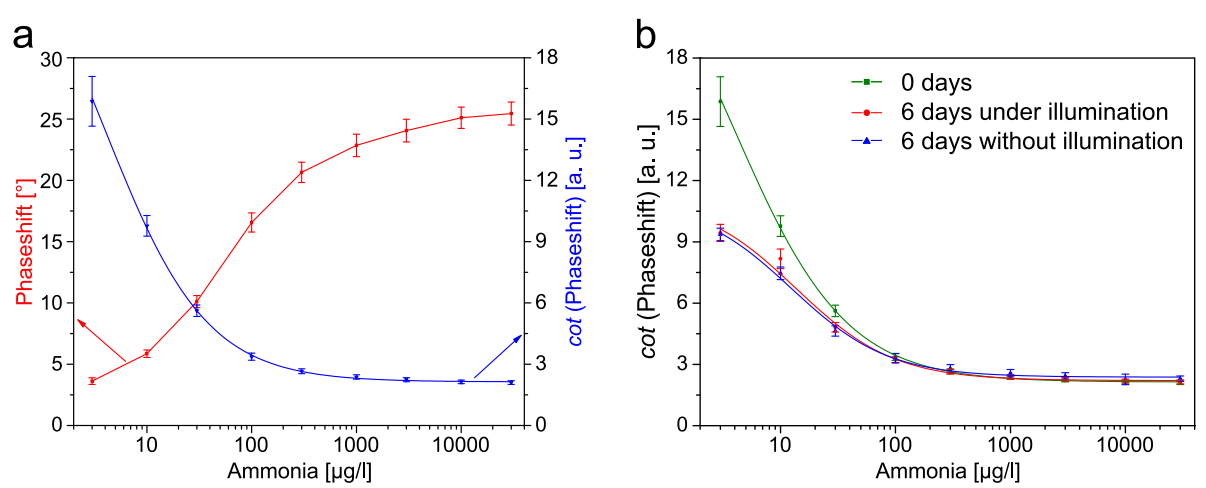

Figure 4 DLR calibration and longtime stability of sensor E. (a) Calibration of sensor E based on phaseshift measurements (red graph mean value of 2 sensor foils) and calculated correlating cotangent values (blue graph); (b) longtime stability comparing illuminated sensors (red graph, mean value of 4 sensor foils) and non-illuminated sensors (blue graph, mean value of 6 sensor foils). 
This ratiometric referencing approach also offers the possibility to use imaging methods [39]. Figure 6 depicts different images of the sensor's surface recorded with a commercially available color camera. The different color channels (red and blue) were separated, analyzed digitally and divided by each other. Both measurement approaches show the same sensor

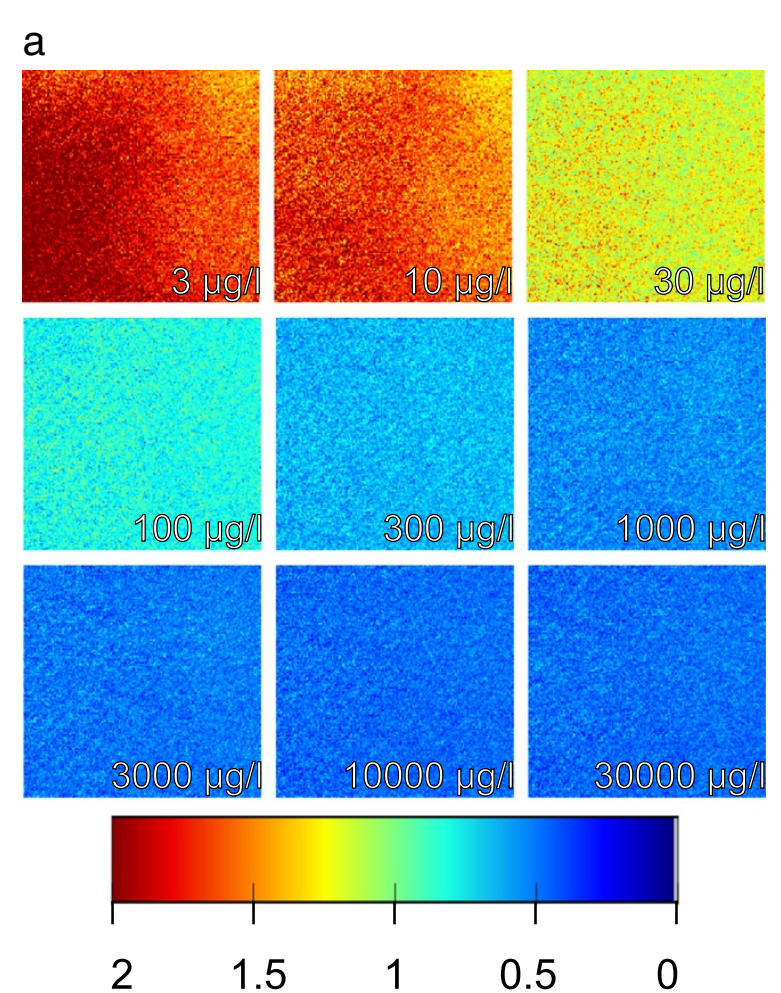

b

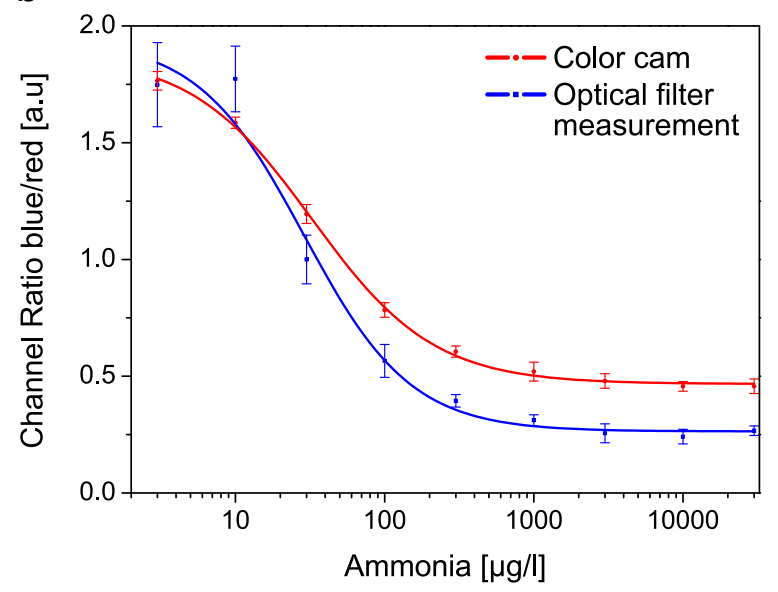

Figure 6 False color imaging pictures and comparison to optical filter measurement. False color imaging pictures (a) taken with a color camera and processed with MathWorks MATLAB (calculation of blue/red channel ratio) and comparison of the mean values of the channel ratio with a calibration carried out with the optical filter measurement approach. measurement in analogy to Figure 4b. Again a drop in fluorescence intensity can be observed independent from the factor of illumination. Despite this drop the sensor itself is still functional and can be used by further recalibration. 
characteristics but differentiate in terms of signal resolution and sensitivity due to different spectral sensitivities of the channels or filters. This shows that this sensor not only can be used for batch measurements but also for imaging and spatial resolution applications, as it has been proven to be useful in marine research by mapping oxygen concentration and $\mathrm{pH}$ distributions via imaging techniques [21].

\section{Experimental \\ Materials}

$1 \mathrm{H}, 5 \mathrm{H}, 11 \mathrm{H}$-[1]benzopyrano[6,7,8,ij]quinolizin-11-one (Coumarin $545 \mathrm{~T}$ or $\mathrm{C} 545 \mathrm{~T})$ and 3-(2-N-Methylbenzimidazolyl)7-N,N-diethylaminocoumarin (Coumarin 30 or C30) were purchased from Sigma Aldrich Chemie GmbH (Steinheim, Germany). 3-(benzothiazol-2-yl)-7-(diethylamino)-2-oxo-2H1-benzopyran-4-carbonitrile (Macrolex Fluorecent Red G or MFR) was purchased from Simon \& Werner $\mathrm{GmbH}$ (Flörsheim am Main, Germany). Bromophenole blue (BPB), Tetraethoxysilane (TEOS) and celluloseacetate (CA) were obtained from Acros Organics (Geel, Belgium). Tetrahydrofuran (THF), N-Cyclohexyl-2-aminoethanesulfonic acid (CHES), acetic acid, sodium dihydrogen phosphate, disodium hydrogen phosphate and sodium chloride were bought from Carl Roth GmbH (Karlruhe, Germany). Hydrogel D1, D4 and D7 were obtained from CardioTech International Inc. (Wilmington, MA, United States). Millipore FHUP Fluoropore $^{\mathrm{TM}}$ Membrane Filter was bought from EMD Millipore Corporation (Billerica, MA, United States). (Ru[dpp $\left.\left.\left(\mathrm{SO}_{3} \mathrm{Na}\right)_{2}\right]_{3}\right) \mathrm{Cl}_{2}$ ) was synthesized in lab following to published results [40].

\section{Synthesis of reference particles}

The reference particles were prepared analogically as reported before [41]. $3.86 \mathrm{~g}$ of TEOS was poured into a solution of $4 \mathrm{ml}$ acetic acid, $1.25 \mathrm{ml}$ and $0,02 \mathrm{mg}$ $\mathrm{Ru}\left(\mathrm{dpp}\left(\mathrm{SO}_{3}\right)_{2}\right)$. The mixture was stirred vigorously right away for 5 minutes and after waiting 30 minutes it was filtered with a Macherey-Nagel MN 619 cellulose filter. The obtained particles were washed two times each with deionized water, ethanol, and acetone. In the end the particle were dried for 24 hours at $80^{\circ} \mathrm{C}$ in a drying oven.

\section{Sensor preparation}

The sensor layers were prepared by coating sensor cocktails onto a Melinex boPET foil (DuPont Teijin Films, Middlesbourgh, UK). Cocktails consisted of the two fluorescent dyes, the indicator, the polymer, and (in case of cocktails for DLR-referenced foils) the particles, dissolved in THF (see Table 1 for sensor cocktail composition). The cocktails were spread onto the foil using a drawdown bar film applicator (wet film thickness $25.4 \mu \mathrm{m}$ ) purchased from BYK-Gardner $\mathrm{GmbH}$
Table 1 Composition of different sensor cocktails (5\%), all dissolved in THF (95\%) (RP: reference particles)

\begin{tabular}{llll}
\hline Sensor & $\begin{array}{l}\text { Host } \\
\text { material }\end{array}$ & FRET-System [\% (v/v)] & $\begin{array}{l}\text { Indicator } \\
\text { [mmol/kg } \\
\text { polymer] }\end{array}$ \\
\hline A & CA & C545T \& MFR [2 / 0.25] & BPB [6] \\
B & D7 & C545T \& MFR [2 / 0.25] & BPB [6] \\
C & D4 & C545T \& MFR [2 / 0.25] & BPB [6] \\
D & D1 & C545T \& MFR [2 / 0.25] & BPB [6] \\
E & D7 \& RP [80:20] & C545T \& MFR [0.4 / 0.2] & BPB [6] \\
F & D7 & C30 \& MFR [2 / 0.2] & BPB [20] \\
\hline
\end{tabular}

(Geretsried, Germany). Immediately after the spreading step, a FHUP Fluoropore ${ }^{\mathrm{TM}}$ membrane filter was laid onto the still wet sensor and pressed down with a brush. The THF evaporates through the filter, leaving a thin hydrogel layer between the membrane filter and the boPET foil (see Figure $7 \mathrm{f}$ for sensor cross section).

\section{Buffer preparation}

$100 \mathrm{mM}$ phosphate buffers ( $\mathrm{pH}$ 7.2) containing different ammonia concentrations were prepared by dissolving sodium dihydrogen phosphate and disodium hydrogen phosphate in water [42]. An equivalent amount of ammonium chloride was dissolved in each buffer, resulting in a free ammonia $\left(\mathrm{pK}_{\mathrm{a}}\right.$ 9.25) concentration calculated by the Henderson-Hasselbach equation (see equation 2).

$$
p H=p K_{a}+\log _{10} \frac{c\left[N H_{3}\right]}{N H_{4}^{+}}
$$

The temperature dependency of the ammoniaammonium equilibrium was calculated by the Gibbs free energy (see equation 3 )

$$
\Delta G=R \cdot T \cdot \ln K
$$

The mathematical compensation of $\mathrm{pK}_{\mathrm{a}}$ of ammonia towards salinity was based on a recently published study by Bell et al [36].

\section{Instrumentation and measurement}

Absorbance spectra were recorded with a Varian Cary 50 UV-VIS spectrophotometer. Luminescence spectra were recorded using a Hitachi F-7000 fluorescence spectrometer. Intensity based and DLR-referenced sensor measurements were carried out with a $\mathrm{pH}-1$ mini (PreSens, Germany). Two wavelength ratiometric measurements were carried out on a SR830 lock-in amplifier from Stanford Research Systems, Inc. (Sunnyvale, CA, United States), a $405 \mathrm{~nm}$ LED from Roithner Lasertechnik (Vienna, Austria) combined with two Hamamatsu photomultiplier tubes and two band pass filters (Carl 


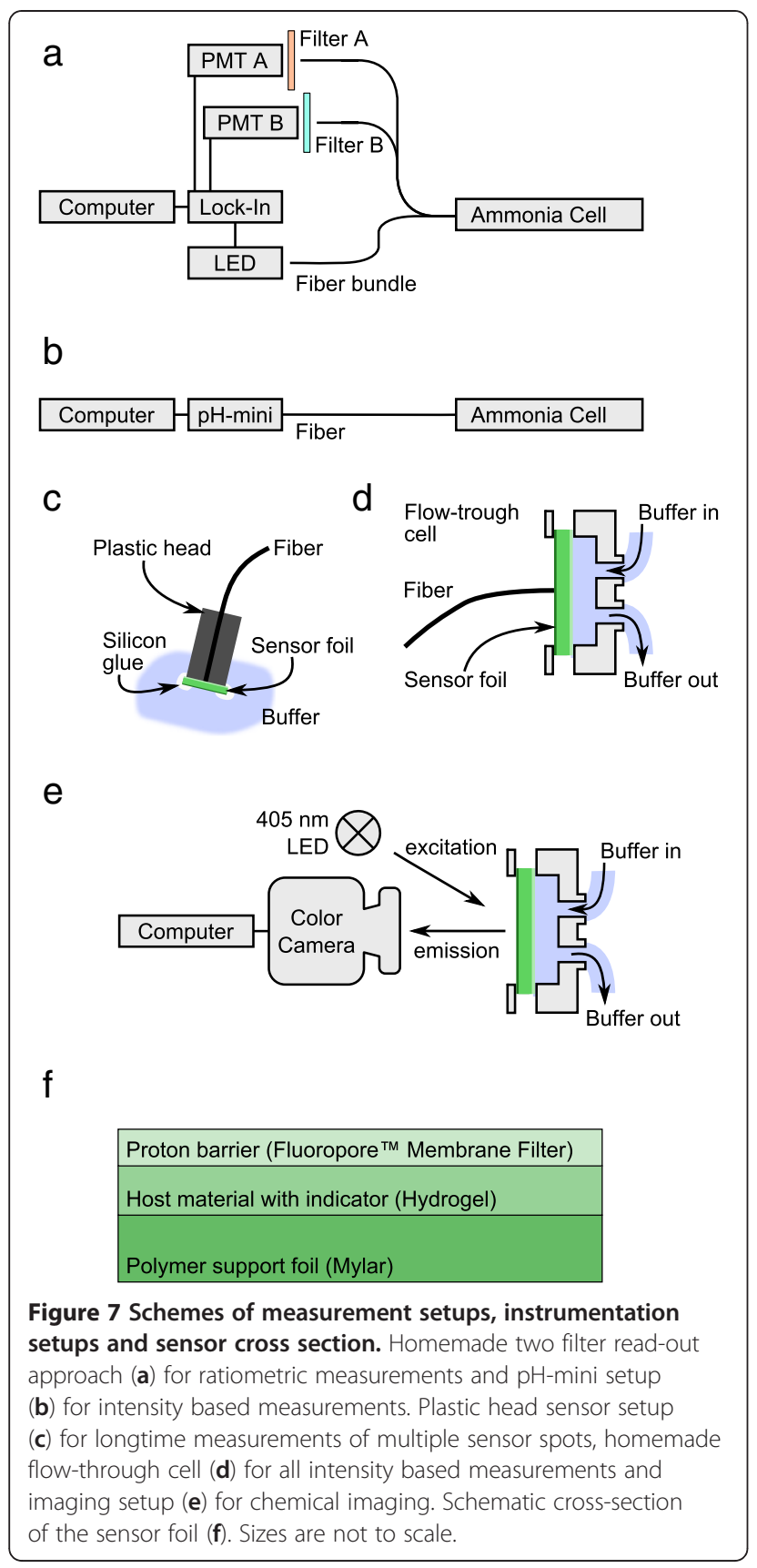

Zeiss 575-625 (575 nm to $625 \mathrm{~nm}$ ) and Horiba Scientific XF $1072(460 \mathrm{~nm}$ to $490 \mathrm{~nm})$ ). Fluorescence imaging was carried out with an F-201C camera from Allied Vision Technologies (Stadtroda, Deutschland). A homemade flow-through cell was used for all intensity based response and calibration measurements calibration measurements. A series of plastic heads (to be plugged on the optical fibers of each readout system) with glued on sensor spots were used for longtime batch measurements. Figure 7 gives an overview over all sensor and instrumentation setups.

\section{Conclusion}

An optical ammonia sensor was developed from commercially available products with simple means of manufacturing. The sensor exhibits a high sensitivity down to almost $1 \mu \mathrm{g} / \mathrm{l}$ ammonia, a response quicker than $120 \mathrm{sec}-$ onds and virtually no cross sensitivity towards $\mathrm{pH}$, temperature and salinity. Two different referencing methods have been presented to demonstrate a simple usability and possible application to imaging technologies.

\section{Abbreviations}

BPB: Bromophenol blue; C30: Coumarin 30; C545T: Coumarin 545T; CA: Cellulose acetate; DLR: Dual lifetime referencing; FRET: Förster resonance energy transfer; MFR: Macrolex Fluorescent Red G; TWR: Two wavelength ratiometric.

\section{Competing interests}

The authors declare that they have no competing interests.

\section{Authors' contributions}

TA planned and carried out the sensor manufacturing, measurements and data evaluation. BU assisted during imaging measurements and data evaluation of said measurements. IK provided scientific advices. TM headed the scientific planning and evaluation of the project. All authors have read and approved the final version.

\section{Authors' information}

All authors are working at the Institute of Analytical Chemistry and Food Chemistry, Graz University of Technology, Stremayrgasse 9, 8010 Graz, Austria.

\section{Acknowledgement}

Financial support by the Austrian Research Promotion Agency (FFG) in the framework of the Austrian Nano Initiative (Research Cluster 0700-ISOTEC) is gratefully acknowledged. We acknowledge Jan Fischer for drawing attention to the publication of Bell et al.

Received: 7 September 2012 Accepted: 24 October 2012

Published: 26 October 2012

\section{References}

1. Mathews CK, Holde KEV: Biochemistry. 2 Sub. 2nd edition. San Francisco: Pearson Education; 1995.

2. Strömberg $N$, Hakonen A: Plasmophore sensitized imaging of ammonia release from biological tissues using optodes. Anal Chim Acta 2011, 704:139-145.

3. Crowley K, Pacquit A, Hayes J, Lau KT, Diamond D: A gas-phase colorimetric sensor for the detection of amine spoilage products in packaged fish. In Proceeding of IEEE Sensor Conference. Edited by Troy Nagle H. 2005:4.

4. Lee DS, Atkins DHF: Atmospheric ammonia emissions from agricultural waste combustion. Geophys Res Lett 1994, 21:281-284.

5. N[otilde]mmik H, Nilsson K-O: Nitrification and movement of anhydrous ammonia in soil. Acta Agriculturae Scandinavica 1963, 13:205-219.

6. Ling Ling T, Ahmad M, Yook Heng L: An amperometric biosensor based on alanine dehydrogenase for the determination of low level of ammonium ion in water. Journal of Sensors 2011, Article ID 980709:1-10.

7. Li QP, Zhang J-Z, Millero FJ, Hansell DA: Continuous colorimetric determination of trace ammonium in seawater with a long-path liquid waveguide capillary cell. Mar Chem 2005, 96:73-85.

8. Müller T, Walter B, Wirtz A, Burkovski A: Ammonium toxicity in bacteria. Curr Microbiol 2006, 52:400-406.

9. Felipo V, Butterworth RF: Neurobiology of ammonia. Prog Neurobio/ 2002, 67:259-279.

10. Timmer B, Olthuis $W$, van den Berg A: Ammonia sensors and their applications-a review. Sensors and Actuators B: Chemical 2005, 107:666-677.

11. Randall D, Tsui TK: Ammonia toxicity in fish. Mar Pollut Bull 2002, 45:17-23. 
12. Krug FJ, Růžička J, Hansen EH: Determination of ammonia in low concentrations with Nessler's reagent by flow injection analysis. Analyst 1979, 104:47

13. Meyerhoff ME, Fraticelli YM, Greenberg JA: Polymer-membrane electrode-based potentiometric sensing of ammonia and carbon dioxide in physiological fluids. Clin Chem 1982, 28:1973-1978.

14. Claps R, Englich FV, Leleux DP, Richter D, Tittel FK, Curl RF: Ammonia detection by use of near-infrared diode-laser-based overtone spectroscopy. Appl Opt 2001, 40:4387-4394.

15. Crowley K, Morrin A, Hernandez A, O'Malley E, Whitten PG, Wallace GG, Smyth MR, Killard AJ: Fabrication of an ammonia gas sensor using inkjet-printed polyaniline nanoparticles. Talanta 2008, 77:710-717.

16. Daridon A, Sequeira M, Pennarun-Thomas G, Dirac H, Krog JP, Gravesen P, Lichtenberg J, Diamond D, Verpoorte E, de Rooij NF: Chemical sensing using an integrated microfluidic system based on the Berthelot reaction. Sensors and Actuators B: Chemical 2001, 76:235-243.

17. Courbat J, Briand D, Wöllenstein J, de Rooij NF: Polymeric foil optical waveguide with inkjet printed gas sensitive film for colorimetric sensing. Sensors and Actuators B: Chemical 2011, 160:910-915.

18. Mills A, Wild L, Chang Q: Plastic colorimetric film sensors for gaseous ammonia. Mikrochim Acta 1995, 121:225-236.

19. Preininger C, Mohr GJ, Klimant I, Wolfbeis OS: Ammonia fluorosensors based on reversible lactonization of polymer-entrapped rhodamine dyes, and the effects of plasticizers. Anal Chim Acta 1996, 334:113-123. 47ref.

20. Förster T: Zwischenmolekulare Energiewanderung und Fluoreszenz. Ann Phys 1948, 437:55-75.

21. Larsen M, Borisov SM, Grunwald B, Klimant I, Glud RN: A simple and inexpensive high resolution color ratiometric planar optode imaging approach: application to oxygen and $\mathrm{pH}$ sensing. Limnology and Oceanography: Methods 2011, 9:348-360

22. Waich K, Borisov S, Mayr T, Klimant I: Dual lifetime referenced trace ammonia sensors. Sensors and Actuators B: Chemical 2009, 139:132-138.

23. Waich K, Mayr T, Klimant I: Fluorescence sensors for trace monitoring of dissolved ammonia. Talanta 2008, 77:66-72.

24. Waich K, Mayr T, Klimant I: Microsensors for detection of ammonia at ppb-concentration levels. Meas Sci Technol 2007, 18:3195-3201.

25. Malins C, Butler TM, MacCraith BD: Influence of the surface polarity of dye-doped sol-gel glass films on optical ammonia sensor response. Thin Solid Films 2000, 368:105-110.

26. Trinkel M, Trettnak W, Reininger F, Benes R, O'Leary P, Wolfbeis OS: Study of the performance of an optochemical sensor for ammonia. Anal Chim Acta 1996, 320:235-243.

27. Chang Q, Sipior J, Lakowicz JR, Rao G: A lifetime-based fluorescence resonance energy transfer sensor for ammonia. Anal Biochem 1995, 232:92-97.

28. Klein R, Voges E: Integrated-optic ammonia sensor. Sensors and Actuators B: Chemical 1993, 11:221-225

29. Mayr T, Borisov SM, Abel T, Enko B, Waich K, Mistlberger G, Klimant I: Light harvesting as a simple and versatile way to enhance brightness of luminescent sensors. Anal Chem 2009, 81:6541-6545.

30. http://www.advbiomaterials.com/products/hydrophilic/HydroMed.pdf.

31. Preininger C, Mohr GJ: Fluorosensors for ammonia using rhodamines immobilized in plasticized poly(vinyl chloride) and in sol-gel; a comparative study. Anal Chim Acta 1997, 342:207-213.

32. Arnold MA, Ostler TJ: Fiber optic ammonia gas sensing probe. Anal Chem 1986, 58:1137-1140

33. http://www.millipore.com/catalogue/item/fhup04700

34. Whelan A, Regan F: Antifouling strategies for marine and riverine sensors. J Environ Monit 2006, 8:880.

35. Waich K, Sandholzer M, Mayr T, Slugove C, Klimant I: A highly flexible polymerization technique to prepare fluorescent nanospheres for trace ammonia detection. Journal of Nanoparticle Research 2010, 12:1095-1100.

36. Bell TG, Johnson MT, Jickells TD, Liss PS: Ammonia/ammonium dissociation coefficient in seawater: a significant numerical correction. Environ Chem 2007, 4:183-186.

37. Borisov SM, Seifner R, Klimant I: A novel planar optical sensor for simultaneous monitoring of oxygen, carbon dioxide, $\mathrm{pH}$ and temperature. Anal Bioanal Chem 2011, 400:2463-2474.

38. Klimant I, Huber C, Liebsch G, Wolfbeis OS: Dual Lifetime Referencing (DLR) - A New Scheme for Converting Fluorescence Intensity into a
Frequency-Domain or Time-Domain Information. In New Trends in Fluorescence Spectroscopy: Applications to Chemical and Life Sciences. 1st edition. Edited by Valeur B, Brochon J-C. Berlin: Springer; 2001:257-274.

39. Schäferling M: The Art of Fluorescence Imaging with Chemical Sensors. Angew Chem Int Ed 2012, 51:3532-3554

40. Castellano FN, Lakowicz JR: A water-soluble luminescence oxygen sensor. Photochem Photobiol 1998, 67:179-183.

41. Karmakar B, De G, Ganguli D: Dense silica microspheres from organic and inorganic acid hydrolysis of TEOS. J Non-Cryst Solids 2000, 272:119-126.

42. Beynon PR, Easterby J: Buffer Solutions. Oxford: Oxford University Press; 1996.

doi:10.1186/1752-153X-6-124

Cite this article as: Abel et al:: Fast responsive, optical trace level ammonia sensor for environmental monitoring. Chemistry Central Journal 2012 6:124.

Publish with ChemistryCentral and every
scientist can read your work free of charge
"Open access provides opportunities to our
colleagues in other parts of the globe, by allowing
anyone to view the content free of charge."
W. Jeffery Hurst, The Hershey Company.
- available free of charge to the entire scientific community
- peer reviewed and published immediately upon acceptance
- cited in PubMed and archived on PubMed Central
- yours - you keep the copyright
submit your manuscript here:
http://www.chemistrycentral.com/manuscript/

